\title{
How Land-use Pattern Influence the Urban Vitality? A Case Study in Shenzhen, China Based on Public Transportation Data
}

\author{
Junyu Ren ${ }^{1,2+}$, Guangzhou $\mathrm{Wu}^{2}$, Hu Huang ${ }^{2}$ \\ ${ }^{1}$ School of Urban Planning and Design, Peking University Shenzhen Graduate School, China \\ ${ }^{2}$ The Smart City Research Institute of China Electronics Technology Group Corporation, China
}

\begin{abstract}
Rapid urbanization has brought about many "urban diseases" in China, such as separation of the workplace and residence, traffic congestion, lack of public spaces, reduction in sense of urban space acquisition, which results in the lower urban space quality and urban vitality. Reshaping urban vitality and improving urban space quality are gaining much attention in urban planning. In this paper, we explored the spatiotemporal characteristics of residents' travels to reveal the spatial pattern of residence, employment, transportation and entertainment in Shenzhen based on public transportation data. We proposed a new method to evaluate urban vitality to show the spatial distribution in Shenzhen according to Jane Jacobs's notions. The result shows the most dynamic areas of Shenzhen are mainly located on the core areas of Futian, Luohu and Nanshan districts with a trend of decline from the centers to suburbs. Then a regression model was established to discuss the relationship between the built environment and urban vitality by analyzing the effects of land-use type, intensity, and mixture on urban vitality. The analysis suggests that both land-use intensity and mixture play a positive role in urban vitality. More specifically, mixed land use is a key factor affecting the sustainability of urban vitality. In addition, commercial land can bring more urban vitality effects than residential and official land because of the high passenger flow intensity and sustainability.
\end{abstract}

Keywords: Urban diseases; Urban space quality; Urban vitality; Public transportation data; Built environment; Land-use mixture; Sustainability

\section{Introduction}

With the continuous urbanization process and the expansion of urban size in China, the flow of urban population, materials, and information have become more frequent. As a result, the characteristics of residents' activities have become more complex and changeable. However, the rapid urbanization has led many "urban diseases", such as separation of occupation and residence, traffic congestion, lack of public space, reduction in sense of urban space acquisition, which result in the decline of urban space quality and the elimination of urban vitality. How to meet the needs of diverse activities, improve the quality of urban life and reshape the vitality space for citizens are critical issues to be solved but often overlooked in many cities.

A series of theories about urban space design has been proposed [1-4], including diversity theory, public communication theory, space place theory, urban catalyst theory, etc. These theories together point out that mixed spatial functions, small-scale neighborhoods, chronic traffic, and street connectivity, are relevant factors for creating urban vitality space [5-7]. Jane Jacobs believes that urban vitality comes from the diversity of urban lives. The process of intertwining between human activities and living space forms the diversity of cities, which is the source of urban safety and vitality.

The empirical study of urban vitality is carried out in two aspects. On the one hand, the researchers mainly focused on the urban open space at the micro-level, which indicated that the open space area, density,

${ }^{+}$Corresponding author. Tel.: +1521059172; fax: +0755-8255595.

E-mail address: renjunyu09@126.com. 
coefficient, and the spatial enclosure are important indicators for evaluating spatial vitality. On the other hand, big data was used to comprehensively analyzed the spatiotemporal distribution of human activities to discuss the distribution characteristics of urban vitality, including LBS (Location Based Service) social network data, mobile phone signaling data, Internet PoIs (Point of Interest) data, taxi GPS data, and IC card swiping data.

However, most researchers measured the vitality of urban space by the intensity of passenger flow or human density, which ignored the time continuity of passenger flow and resulted in the big deviations. For example, the residential areas, office areas, which often have large-scale passenger flow during peak periods, with the limited passenger flow in other times, are not dynamic areas. Moreover, Jane Jacobs also suggested that "the even distribution of passenger flow over time" is the key to urban vitality. It means that the vitality of urban space depends on a large scale of passenger flow and passenger flow at different times.

This study focused on studying the quality of urban space and attempted to identify the areas which lack of vitality in Shenzhen. We analyzed the general rules of residents' travel and constructed a new method to comprehensively evaluate urban vitality of different areas with the big data of urban public transportation in Shenzhen, including taxi GPS data, bus GPS data, and IC card swiping data. And then, we established a regression model to discuss the relationship between the built environment and urban vitality by analyzing the effects of land use type, intensity, and mixture on urban vitality. The research results provide a theoretical basis for better urban planning and development in the future.

\section{A Review of Relevant Studies}

Urban vitality usually refers to the capacity of a place to induce lively social and economic activities [8]. It is difficult to well understand the meaning of urban vitality because of its rich interpretations and representations. It depends on the gathering of "quantity, density and heterogeneity of people". Urban vitality connotes the immaterial city lives of diversity, vitality, and attractiveness. The great urban vitality depends on the physical entities of urban form. The area with a better-built environment is more likely to gather several human activities, which makes it a dynamic place [9-10]. In other words, urban vitality is a social process that has strong ties with urban form [11-12]. Some researchers have done some work to explore how morphological features produce diverse and vibrant urban lives [13-14].

Urban morphology focuses on the physical aspects of the built environment, including streets, blocks, plots, and buildings as well as the transformative processes that shape these key elements [14-16]. Urban design is closely related to urban morphology, which is usually used to analyze, organize and shape urban structure and functions for urban space. A good urban design is useful to make good quality urban space with high vitality [17-18]. Some theories about promoting urban vitality by urban design have been discussed [5, 19]. The street accessibility, building density and typology, and diversity are frequently recognized as the most important physical underpinnings of urban vitality.

Otherwise, some empirical studies do concerns about human activities. They use new urban data, including Taxi GPS data, Location-based data, Check-in data, Smartphone data, etc., to analyze the human activities to distinguish the hot areas. Some of the new urban data of built environment help to gather information about urban form and function in a relatively automatic, accurate and consistent manner [5], which is helpful to make an excellent analysis of urban morphology [20-21]. For instance, OpenStreetMap (OSM) provides open information about large geographic areas with high spatial accuracy [22]. Points of Interest (PoIs) and checkin data from social media platforms provide new ways of gauging how people feel and enjoy urban places [23].

Many systematic literature reviews have tried to summarize and generalize the association between the physical environment and human activity [24-27]. A built environment that consists of high-density and mixed land uses, as well as pedestrian-friendly street networks with high street connectivity can encourage walking and other physical activities [28-30]. Public open spaces such as parks and greenways take a big impact on human activities [30-31].

Jane Jacobs (1961) argued that expressway construction and large-scale urban redevelopment projects led to less walking activity, and therefore killed urban vitality. She suggested that a mix of land uses, small block sizes, coexistence between old and new buildings within a district, high developmental density were the key conditions that must be met. Moreover, Jacobs points out that the highly dynamic areas do have large intensive 
human activities and the activities are sustainable over time. According to her notions, our research tries to construct a method to measure the urban vitality and further discussed how the built environment influences the urban vitality. The study results could help the advocacy planning movement and reshape the dynamic urban space.

\section{Data and Methods}

\subsection{Study Area}

In this paper, we focus on Shenzhen, one of the largest and fastest growing cities in China. Shenzhen is a special economic zone (SEZ) in China and the number of residents has since rapidly increased more than forty times. The urban built-up area has also expanded significantly to accommodate rapid population growth with urban issues as emerging in some big cities of developed countries, which makes it a good sample to analyze the urban disease.

Shenzhen was built from scratch in the past four decades. The city has accumulated various urban forms that are typical in post-reform China, e.g., urban villages and gated residential neighborhoods[32-34]. Shenzhen consists of four inner-city (Luohu, Futian, Yantian, and Nanshan) and six outlying districts (Baoan, Longhua, Longgang, Guangming, Pingshan, and Dapeng) with 74 Sub-district Offices as shown in Figure 1. Luohu and Futian districts are part of the initial special economic center and have undergone the longest development process. Nanshan districts is a gathering area for high-tech enterprises, which makes Nanshan a core area for future development. Therefore, these districts are the main analysis area in our study. In addition, we have more complete data of these districts to support this research, including Points of Interest, Land use data, and transportation facilities.

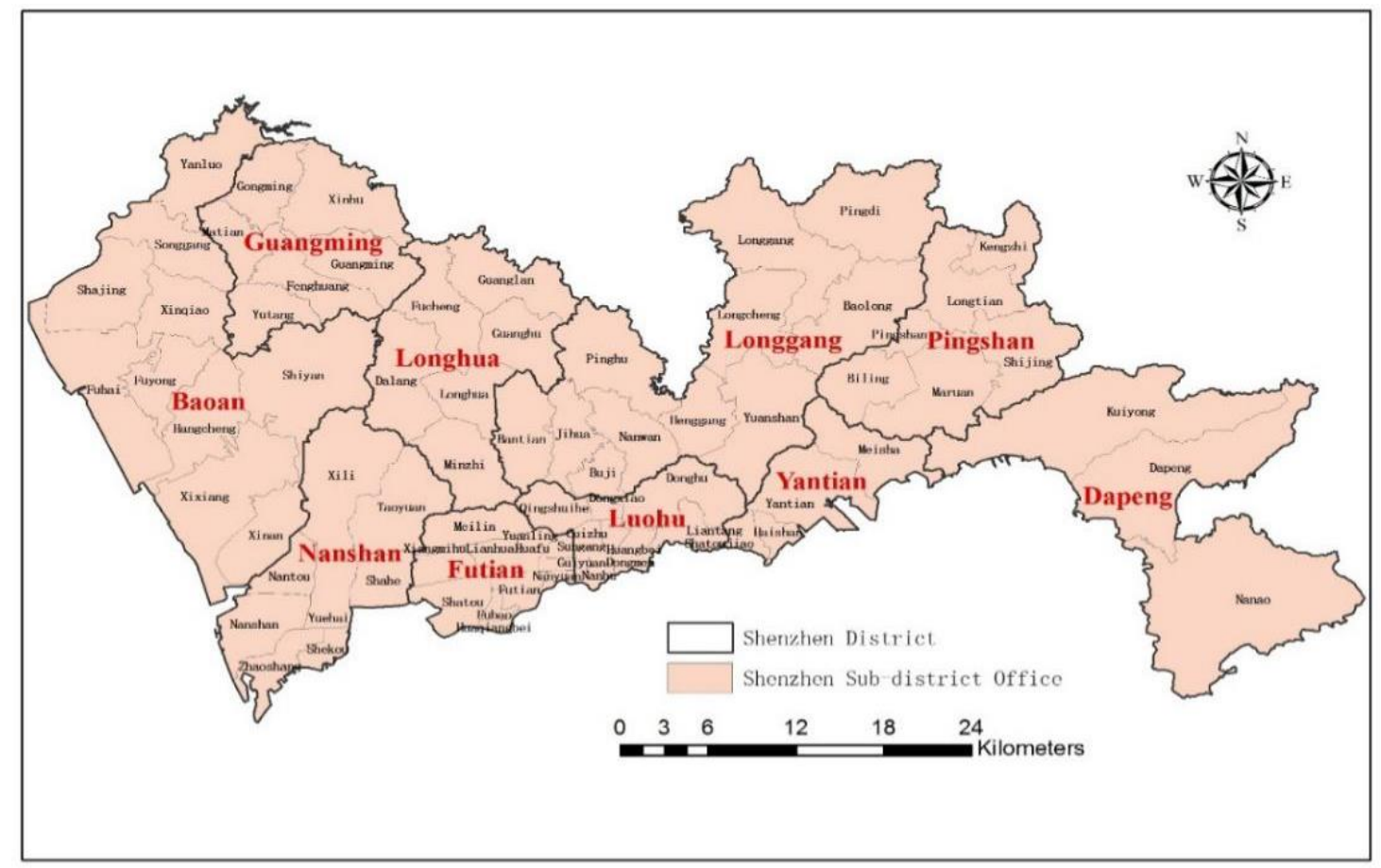

Fig. 1: Shenzhen District and Sub-district Office

The basic research data includes taxi GPS data, bus GPS data, and IC card swiping data of 5 working days in Shenzhen from 2018.3.26 2018.3.30. To more accurately measure the spatial distribution of urban vitality, we first divided the administrative division map of Shenzhen into 54186 grids with the size of $200 \mathrm{~m} * 200 \mathrm{~m}$, as the basic research units. Then, we extracted the OD points of public transportation from the basic data and matched them to the specific grids with the gravity model. Thus, we can analyze the distribution characteristics of passenger flow in different grids and quantitatively evaluate the spatial vitality of different areas in the city.

\subsection{Methods}

Jane Jacobs's (1961) notions about urban vitality are "Uniform distribution of human flow over time". The 
vitality of an area depends on two aspects, including sufficient passenger flow and passenger flow sustainability. The area where people only arrive at a certain period is not dynamic, such as residential areas and official areas which tend to have many passenger flow at peak times but very limited at others.

Therefore, we constructed a new method to achieve a more comprehensive assessment of urban vitality based on passenger flow intensity and sustainability, as follows:

1. Passenger flow intensity: the total number of passengers in research units.

$$
V_{1 i}=f_{i}=\sum_{t} f_{i t}
$$

Where $f_{i t}$ is the total amount of passengers in the grid $\boldsymbol{i}$ during the period $\boldsymbol{t}$.

2. Passenger flow sustainability: Shannon entropy of passenger flow distribution.

Shannon entropy represents the uncertainty of a random distribution, which is used to measure the diversity of random distributions. The larger the Shannon entropy of a random distribution, the more diversity of the distribution. In this study, if the Shannon entropy of passenger flow distribution over time is larger, it means that passenger flow sustainability is higher.

$$
V_{2 i}=-\sum_{t} p_{i t} \ln p_{i t} \quad p_{i t}=\frac{f_{i t}}{\sum_{t} f_{i t}}
$$

Where $\boldsymbol{p}_{i t}$ is the proportion of the passenger flow in the research grid $\boldsymbol{i}$ during the period $\boldsymbol{t}$.

Therefore, urban space vitality is expressed as follows:

$$
V_{i}=V_{1 i} \times V_{2 i}=f_{i} \times\left\lfloor-\sum_{t} p_{i t} \log \left(p_{i t}\right)\right\rfloor=-\sum_{t} f_{i t} \log \left(p_{i t}\right)
$$

The built environment data were spatially matched to the specific units, and the building data in each unit was quantitatively described, including land use type, intensity, and mixture, i.e. Besides that, some control variables also were added into the regression model, such as transportation facilities, traffic accessibility, location conditions, etc. The regression model as follows:

$$
V_{i}=\beta_{0}+\sum_{j}^{\prime} \beta_{j} X_{i j}+u
$$

Where $\boldsymbol{B}_{0} \ldots \boldsymbol{B}_{j}$ are the parameter to be estimated, $\boldsymbol{X}_{i j}$ is the built environment variable $j$ of unit $i$.

\section{Residents' Travel Characteristics}

\subsection{General Characteristics of Residents' Travels}

Residents' travels have prominent characteristics on weekdays, where the proportion of passenger flow in peak hours accounts for $50.54 \%$. The passenger flow intensity of peak hours in the morning is higher than the evening, while the evening rush period is longer, as shown in Figure 2.

In contrast, taxi passenger flow has no obvious commuting difference between peak and off-peak hours. The highest taxi flow intensity occurs between 14:00 and 16:00, and then it maintains a stable level. Therefore, taxi travel mode is more likely for residents' non-commuting travel.

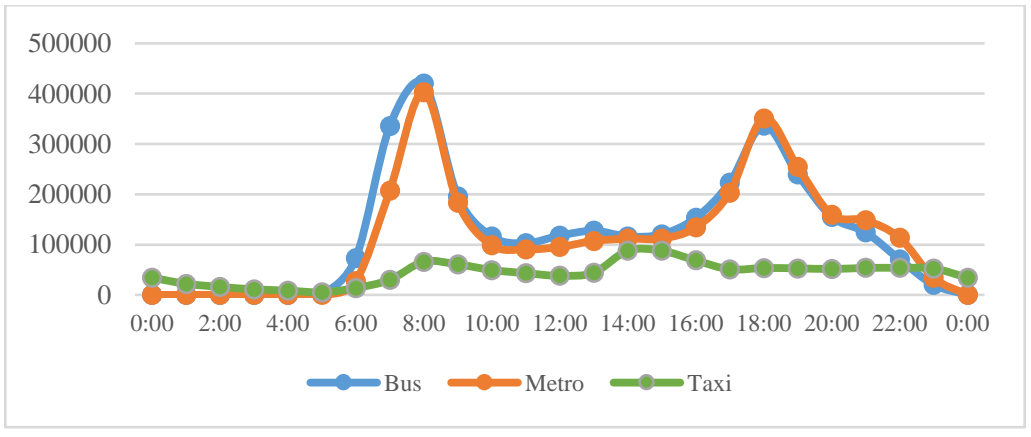

Fig. 2: Different modes Passenger flow distribution in Shenzhen 
The distribution of public transport passenger flow has obvious directionality. The commuter passenger flow in the peak periods of Shenzhen mainly divided into three types as Internal commuting of City center, Internal Commuting of City suburbs and External Commuting between city suburbs to center. The passenger flow in off-peak hours mainly concentrates near the place of residence, and the proportion of long-distance and cross-regional travels is relatively small.

\subsubsection{Characteristics of Residents' Travel in Peak Hours}

Residents' travel characteristics of public transportation are following the tidal law. The passenger flow of peak hours can mainly divide into three types as follows:

(1). Internal Commuting of City Center: Internal Commuting of City center is mainly concentrated in Nanshan District (Yuehai and Nantou sub-district office), Futian District (Futian, Huafu, and Huaqiangbei subdistrict office), Luohu District (Nanhu, Dongmen, and Guiyuan sub-district office) as shown in Figure 3. These areas have formed a fixed occupation and residence spatial pattern with a high level of economic development, a large number of employment opportunities, and a corresponding residential area.

(2). Internal Commuting of City Suburbs: Internal Commuting of City suburbs mainly takes place at Longhua, Bantian, Minzhi, Buji, and Xin'an sub-district office. There are a large number of labor-intensive industrial areas and residence with low living costs (Urban Village), in city suburbs. This special occupation and residence structure satisfies the choice of many migrant workers for employment and residence, which brings a large number of migrants to these areas and forms a spatial pattern in which the employed people live nearby.

(3). External Commuting Between City Suburbs to Center: External Commuting between city suburbs to the center is mainly from Longhua, Baoan and Longgang district to Futian, Nanshan and Luohu district. With the development of the economy and the expansion of the city, the scope of employment influence in the core area of the city center has gradually spread to city suburbs, which attracts a number of employers from city suburbs. Considering the high cost of living in the central area and the relatively lower cost in suburbs, some of the inhabitants choose to move to city suburbs, resulting in a typical spatial pattern of living in suburbs and working in city centers.

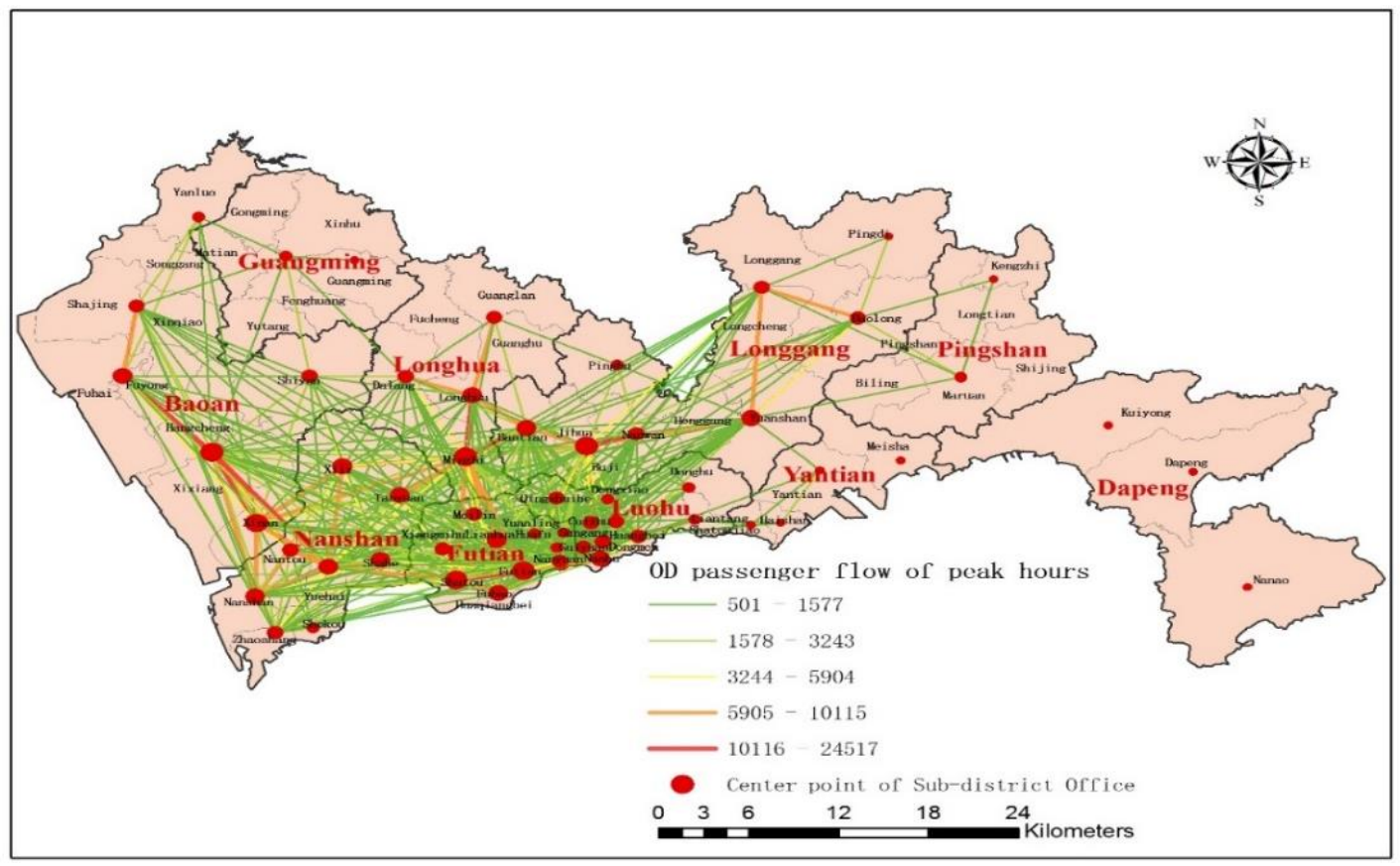

Fig. 3: OD map of passenger flow during the peak period

\subsubsection{Characteristics of Residents' Travel in Off-peak Hours}

During off-peak hours (9:00 17:00), Futian and Luohu have a large number of travels, while others are relatively small, which is consistent with the current land use layout and population distribution in Shenzhen. It can be seen from the OD map as shown in Figure 4, in which most residents take the short-distance travel 
because their daily leisure activities are concentrated around the residence, the proportion of long-distance and cross-region travels is relatively small, which is also in line with the habits of ordinary residents.

Futian and Luohu districts have the highest population density in Shenzhen, well-developed service and retail industry, well-equipped public facilities. As a result, there are a large number of travels for leisure and entertainment during off-peak hours. Nanshan District has a relatively lower population density with a higher proportion of the employed population because of the clustered high-tech enterprises. Therefore, the passenger flow in the off-peak period is less than Futian and Luohu districts.

Due to the low proportion of non-employed people in the suburbs area, the tertiary industry is relatively undeveloped, the retail sales of social consumer goods are generally lower, and the public infrastructure facilities are insufficient. All these result in the small number of travels in off-peak hours with some residents leaving for central areas.

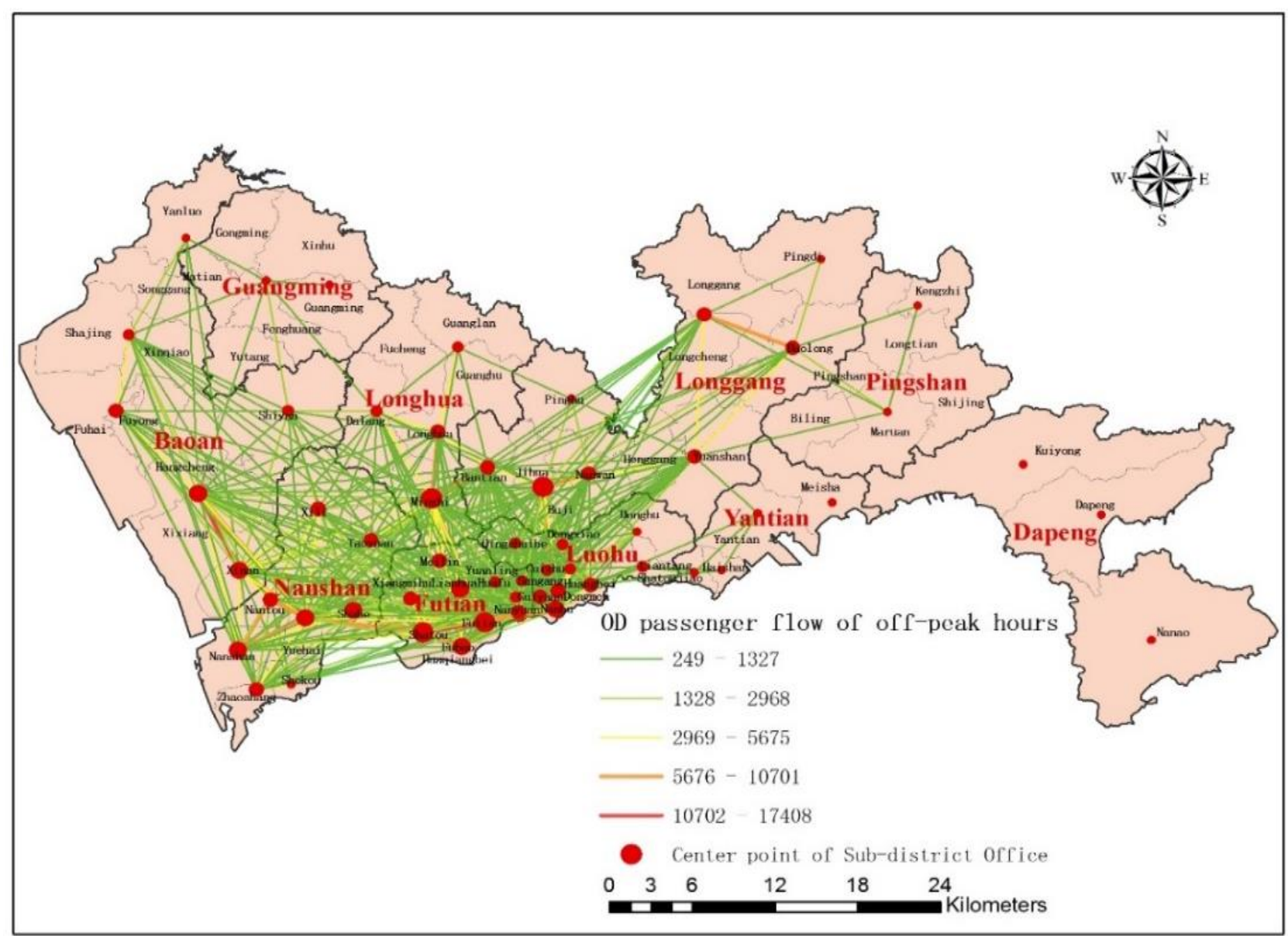

Fig. 4: OD map of passenger flow during the off-peak period

\subsection{Efficiency of Rail Transit}

Rail transit has become an important part of the urban transportation system with its strong passenger capacity. The proportion of commuter passengers by the metro between suburbs and centers during the peak period is relatively high. Minle, Xili, Baishizhou, etc., which are densely populated are mainly commuting outside. On the contrary, Huaqiangbei, Convention and Exhibition Center, Science and Technology Park, etc., which have rich employments, are mainly commuting inside.

The passenger efficiency of rail transit system plays a vital role in urban public transportation services, which can help improve the economic benefits of the rail transit system. According to the OD points of rail transit passenger flow, we combined with shortest path principle to identify the travel trajectory of different ODs and calculate the cross-section passenger flow of different stations and lines, as shown in Figure 5. 


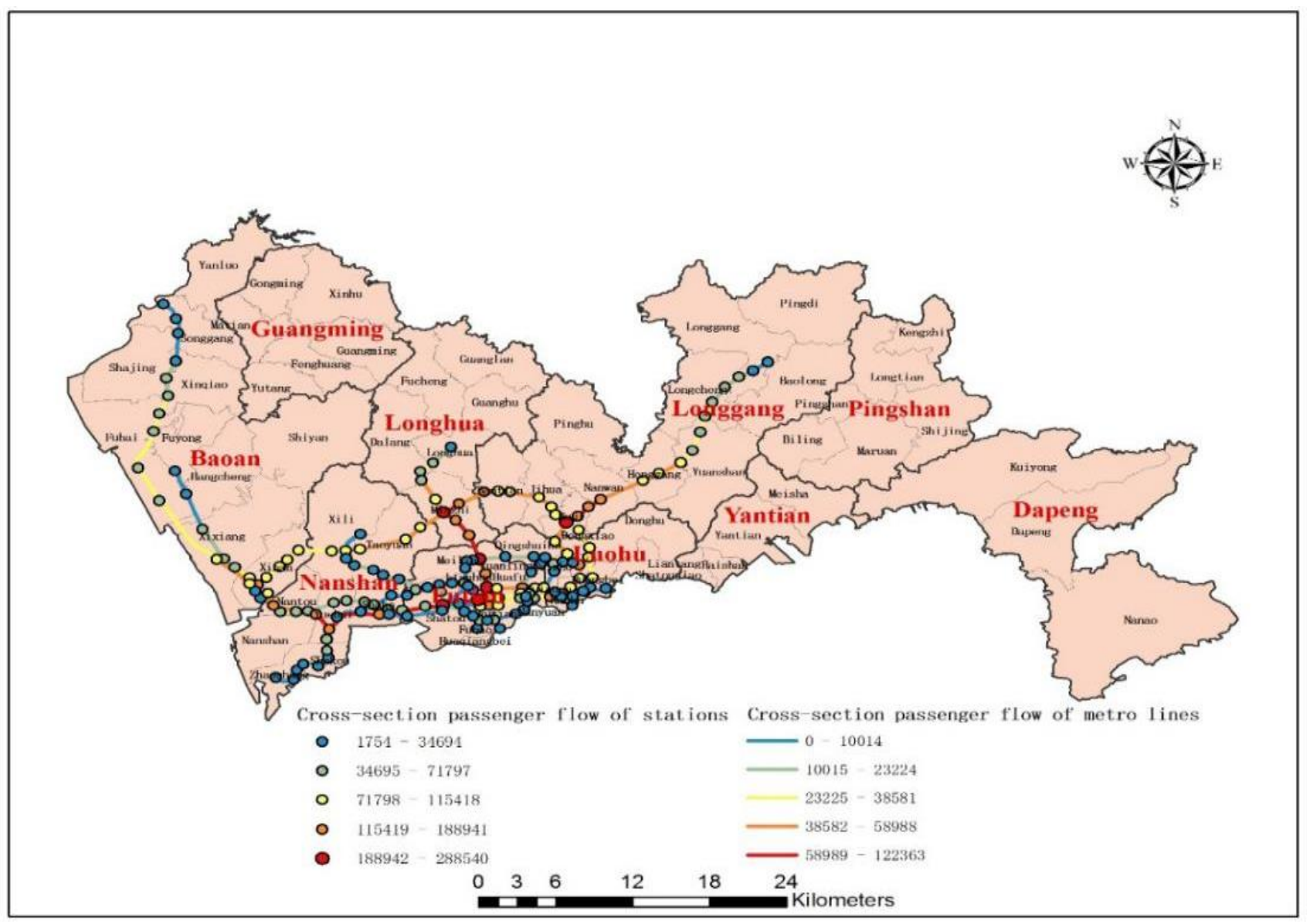

Fig. 5: Cross-section passenger flow distribution of rail transit stations and lines

Metro Line 4 has the highest transportation efficiency. Either nodes or the cross-section passenger flow of Line 4 is highest. Because in recent years, a large number of residential areas have been built in the Longhua area resulting in the highest commuting needs.

Metro Line 4, Line 3 and Line 11 are the main lines connecting city centers and suburbs carrying a large number of passengers from suburbs to central areas. It well meets the residents' need for long-distance travel demand because of its fast and efficient transportation serviceability. Metro Line 5 is mainly for connecting the suburban areas. It passes many densely populated areas including Xili, Minzhi, Wuhe, etc., and transportation hubs (Shenzhen North Station and Buji Station). Thus, the passenger transportation efficiency is relatively high.

Metro Line 2, Line 7 and Line 9 are all rail lines connecting the central areas. The passenger transportation efficiency is relatively low, with only a few transfer stations have a large number of passengers. Because the coverage of buses in central areas is higher. A large number of residents may choose buses for their shortdistance travel, which reduces the demand for long-distance travel services of rail transit.

\subsection{Separation of Occupation and Residence}

"Residence" and "employment" are two key variables in the evolution of urban spatial structure. The spatial distribution of residence and employment affect the spatiotemporal distribution characteristics of urban residents' commuting. The separation of occupation and residence has severely restricted the sustainable development of cities and the quality of citizens' lives. This paper quantitatively evaluated the separation degree of occupation and residence in different areas based on the characteristics of residents' travel during the morning peak period. The "internal and external commuting ratio" reflects the self-sufficiency of residence and employment, as follows: 


$$
\psi(K)=\frac{F_{K, K}}{\sum_{I \neq k}\left(F_{K, I}+F_{I, K}\right)}
$$

Where $F_{K, K}$ is the number of internal working trips in the region $K$ and $F_{K, I}$ is the number of external working trips from region $K$ to region $I$.

Shenzhen has an obvious separation of occupation and residence as shown in Figure 6 and Figure 7. The "internal and external commuting ratio" in Yantian, Luohu, Futian and Nanshan district is relatively lower, which means the degree of separation of occupation and residence is higher.

The separation of occupation and residence in Yantian is most serious. The positioning of the Yantian District is modern tourism and harbor region. The tertiary industry is mainly based on the tourism service industry, lacking the high-tech industry, which makes the employment opportunities insufficient.

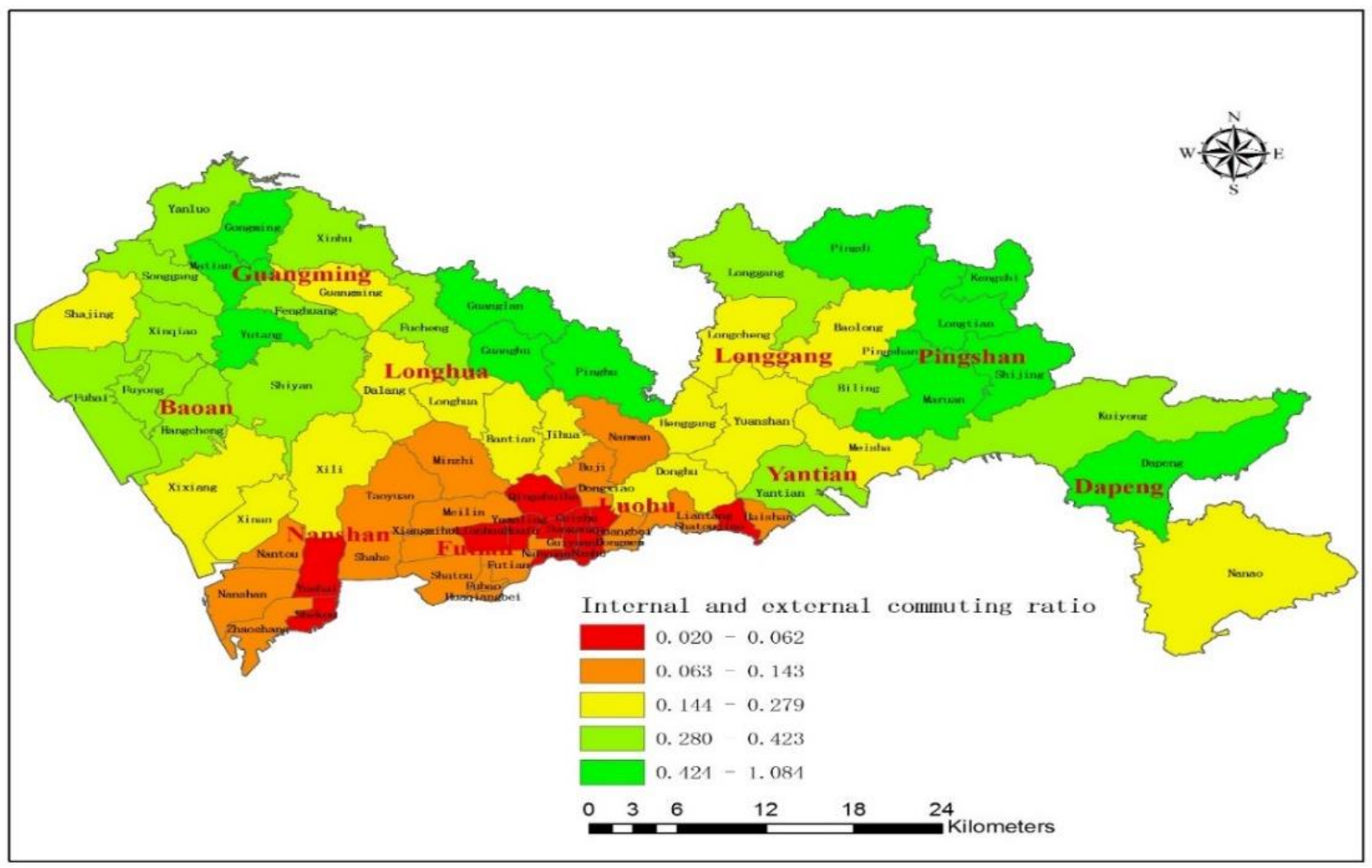

Fig. 6: The sub-districts' "internal and external commuting ratio" during the peak period in the morning

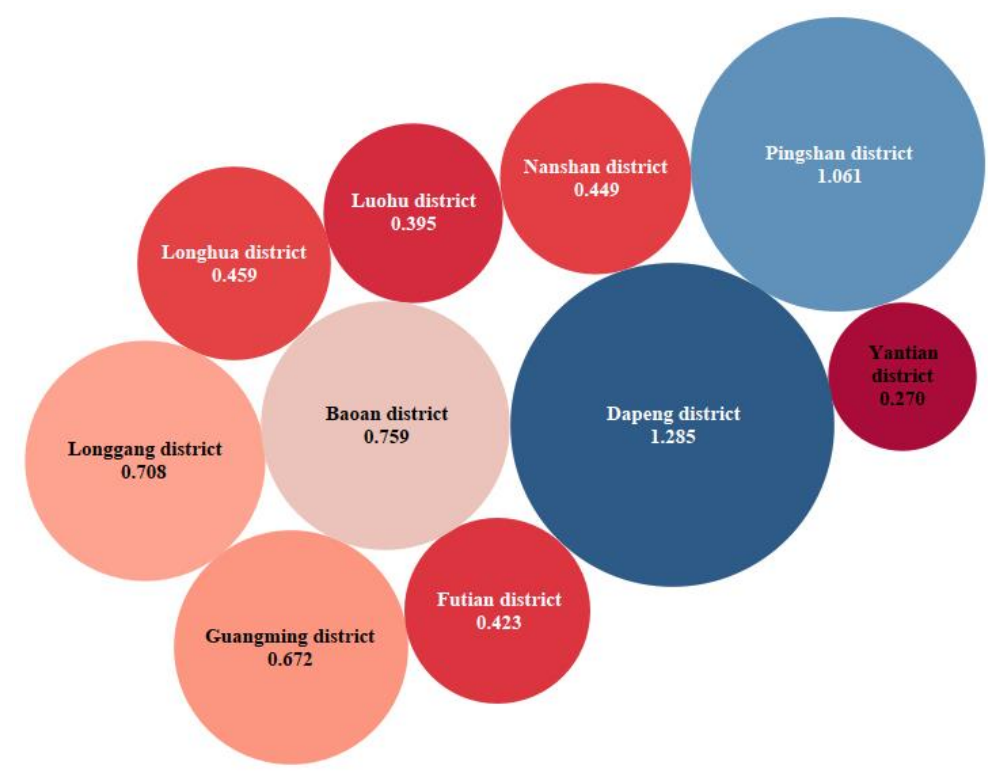

Fig. 7: The districts' "internal and external commuting ratio" during the peak period in the morning 


\subsection{Urban Space Vitality Assessment}

The city is the carrier of citizens' lives, and human activities are the most direct source of urban vitality. According to the equation (3), the vitality value of each grid can be calculated, and then the hotspot region of urban vitality was identified by spatial nuclear density analysis method, as shown in Figure 8.

The most dynamic areas in Shenzhen are Futian, Luohu and the core area of Nanshan districts because of the long history of development. Shenzhen is a typical city of TOD development. Rail transit plays an important role in the development of Shenzhen. Areas around the metro station and line are more dynamic than others.

In recent years, with the adjustment of Shenzhen's industrial structure, some industries have been transferred to the suburbs. Affected by traffic conditions especially the rail transit, the areas closer to the city centers, such as Baoan, Longhua, and Longgang, get a much higher level of development than other suburban areas, which results in the greater urban vitality.

Yantian, Guangming, Pingshan, Dapeng district and the northern part of Bao'an district are far away from the city center with the poor public transportation. They cannot attract more people to working or living there, which makes the lower urban vitality.

The comparison of vitality among different street offices (Figure 9) shows that the Minzhi, Xin'an, Buji, Xixiang, and Bantian sub-district office have the best distribution of passenger flow, with the higher passenger flow intensity and passenger flow sustainability (I-Quadrant). Due to the relatively balanced occupational and residential space in these areas, there will not be a large number of inflows or outflows during peak periods. As a result, the difference in passenger flow between the peak hours and the off-peak period is relatively small.

In contrast, Futian, Nanhu, Yuehai, Huangbei sub-district office, etc., have much higher passenger flow intensity with the lower time sustainability (IV-Quadrant), which is because there are a large number of resident flows during peak hours, with the extremely low internal and external commuting ratios.

Although the sub-district offices of Guangming, Dapeng, and Pingshan District have a high passenger flow sustainability, their passenger flow density is low (II-Quadrant), which makes the urban vitality is insufficient.

Yantian District is lacking urban vitality because of the lowest of both passenger flow density and the passenger flow are sustainability (III-Quadrant).

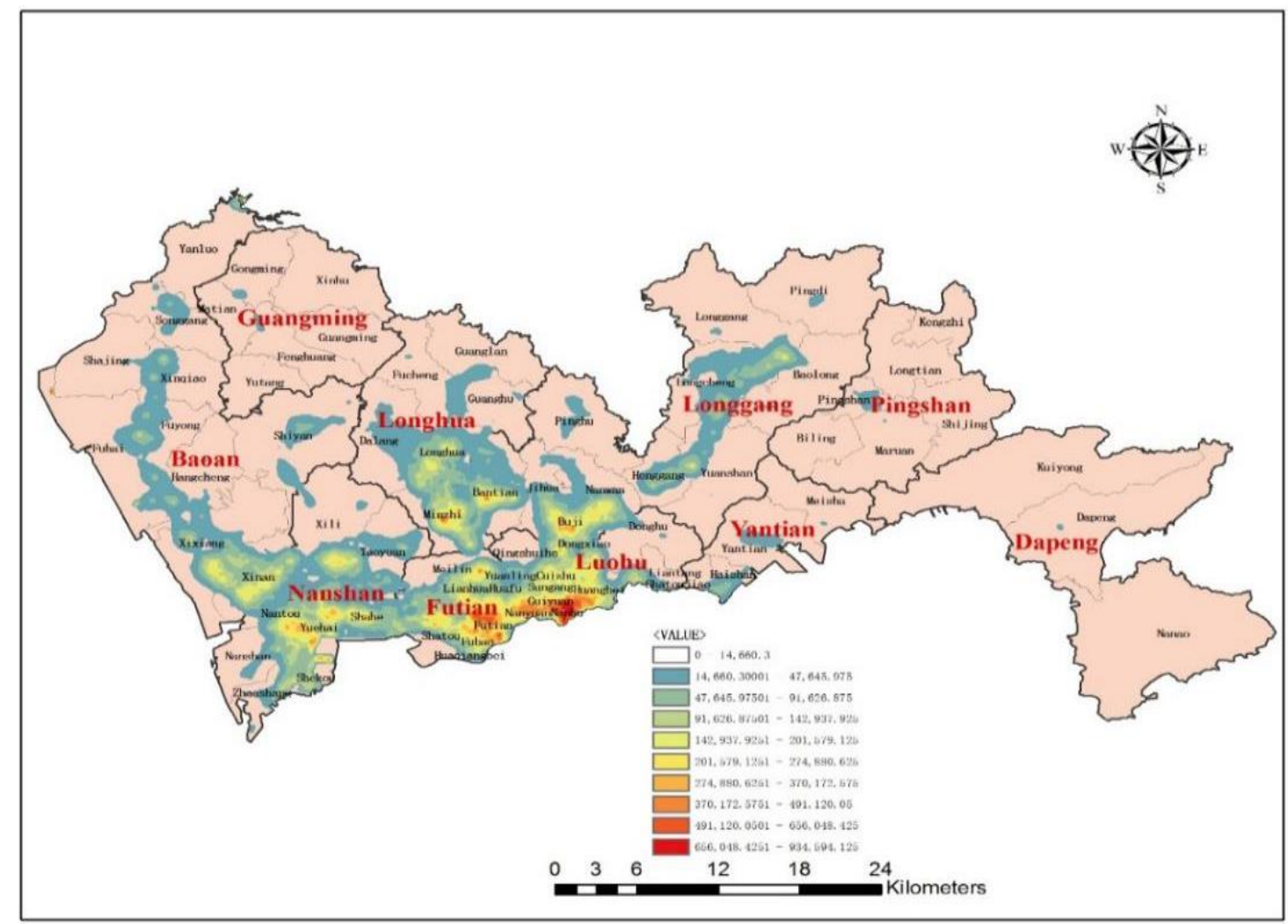

Fig. 8: Urban vitality spatial distribution in Shenzhen 


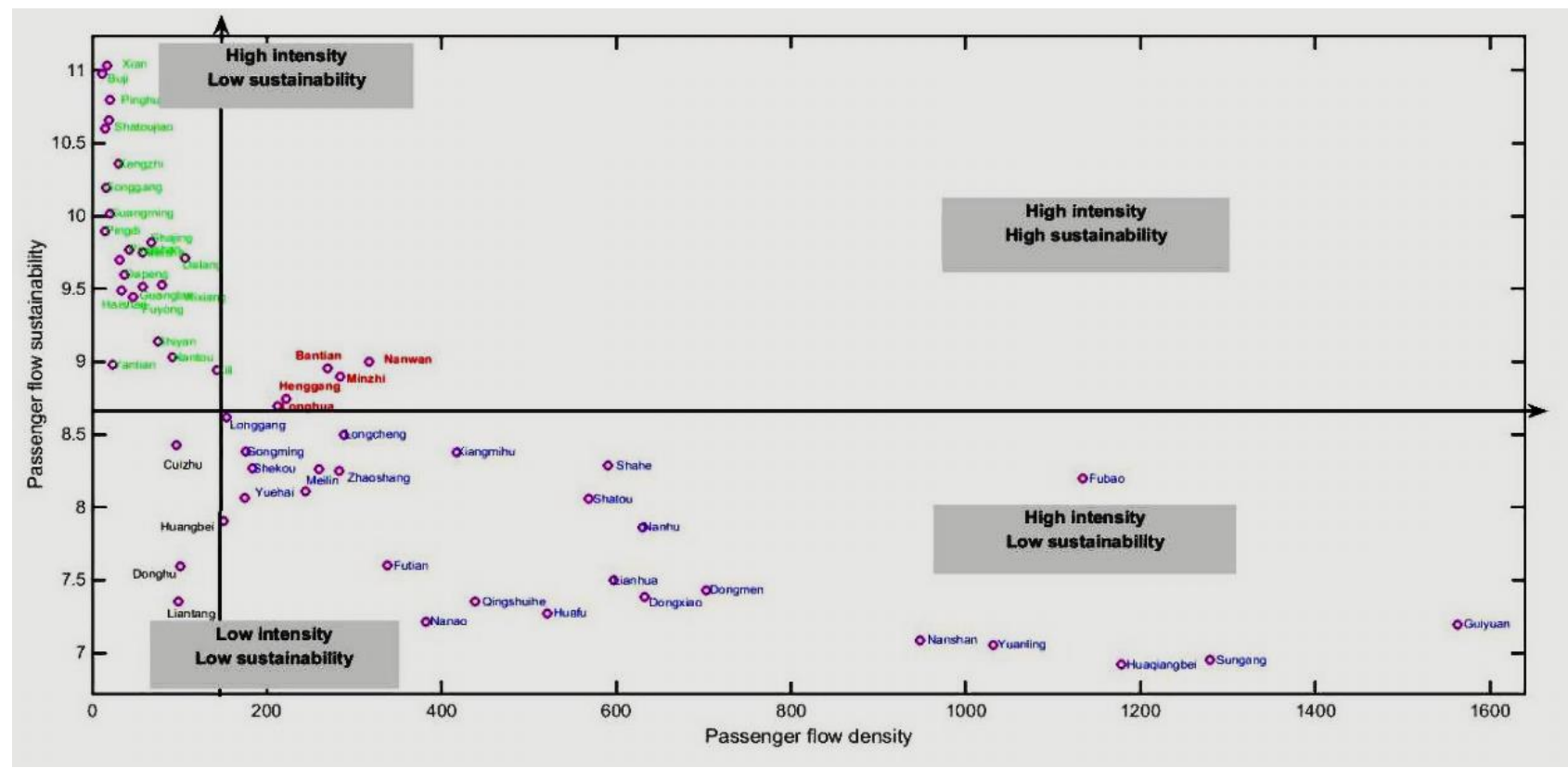

Fig. 9: Urban vitality distribution of Sub-district Office

\section{Analysis of the Relationship Between the Built Environment and Urban Vitality}

The city is the carrier of human lives, and human activities are the most direct source of urban vitality. Jane Jacobs points out that urban essence lies in diversity. The vitality of urban life and social economy depends on the comprehensiveness and mixture of urban functions rather than its unity. The purpose of urban planning is to foster and coordinate multiple functions to meet the diverse and complex needs of different groups of people. The highly mixed urban area is often the most dynamic and attractive area in the city. In this section, we would further explore the relationship between land use and urban vitality, and then a regression model was built to analyze how different land use patterns influence the urban vitality.

Firstly, we need to expand the basic research unit instead of the original grid. A spatial clustering method through the spatial distribution characteristics of urban vitality was used to combine some grids into a larger research unit. According to the results of clustering, the built environment data was matched with the new research unit. Before building the regression model, we need to descriptively analyze the data to enhance the understanding of the correlation between independent variables and dependent variables.

Table 1: Description of land use variables

\begin{tabular}{|c|c|c|}
\hline Land use type & Variables & Description \\
\hline Residential land & $\mathrm{L}_{\mathrm{R}}$ & Mainly including residential quarters, apartments and living facilities \\
\hline Private house & LPH & Urban village \\
\hline Commercial land & $\mathrm{L}_{\mathrm{C}}$ & $\begin{array}{l}\text { Mainly including commercial pedestrian streets, retail stores, service } \\
\text { industries, shopping malls, etc. }\end{array}$ \\
\hline Public land & $\mathrm{L}_{\mathrm{P}}$ & Mainly including public infrastructure, parks, and plazas \\
\hline Official land & Lo & Mainly including government and commercial office buildings \\
\hline Industrial land & $\mathrm{L}_{\mathrm{I}}$ & Mainly including industrial factory and industrial parks \\
\hline Municipal land & $\mathrm{L}_{\mathrm{M}}$ & $\begin{array}{l}\text { Mainly including municipal infrastructure, such as gas stations, parking lots, } \\
\text { public toilets, etc. }\end{array}$ \\
\hline Others & Lot & Others except the above land use types \\
\hline
\end{tabular}

Land use variables mainly include building area (Table 1). Different types of land use have different impacts on urban vitality. As seen in Figure 10 and Figure 11, there is a significant correlation between land use and urban vitality, except the industrial land. Among them, the correlation between residential, commercial, 
official, public land, and urban vitality are stronger, and the correlation coefficient is more than 0.5 . Private House and Municipal land have a weaker influence on urban vitality. In addition, mixed land use also has a significant positive on urban vitality, where the correlation coefficient is 0.32 . The transportation system plays an important role in urban spatial structure and human activities. Areas with convenient transportation are usually taking a high level of development, which can attract more citizens to make a number of activities and create a high-quality urban space. As shown in Figure 12, transportation system ability including bus, metro, road network, shared bicycle, take a positive impact on urban vitality with the correlation coefficient nearing to 0.5 .
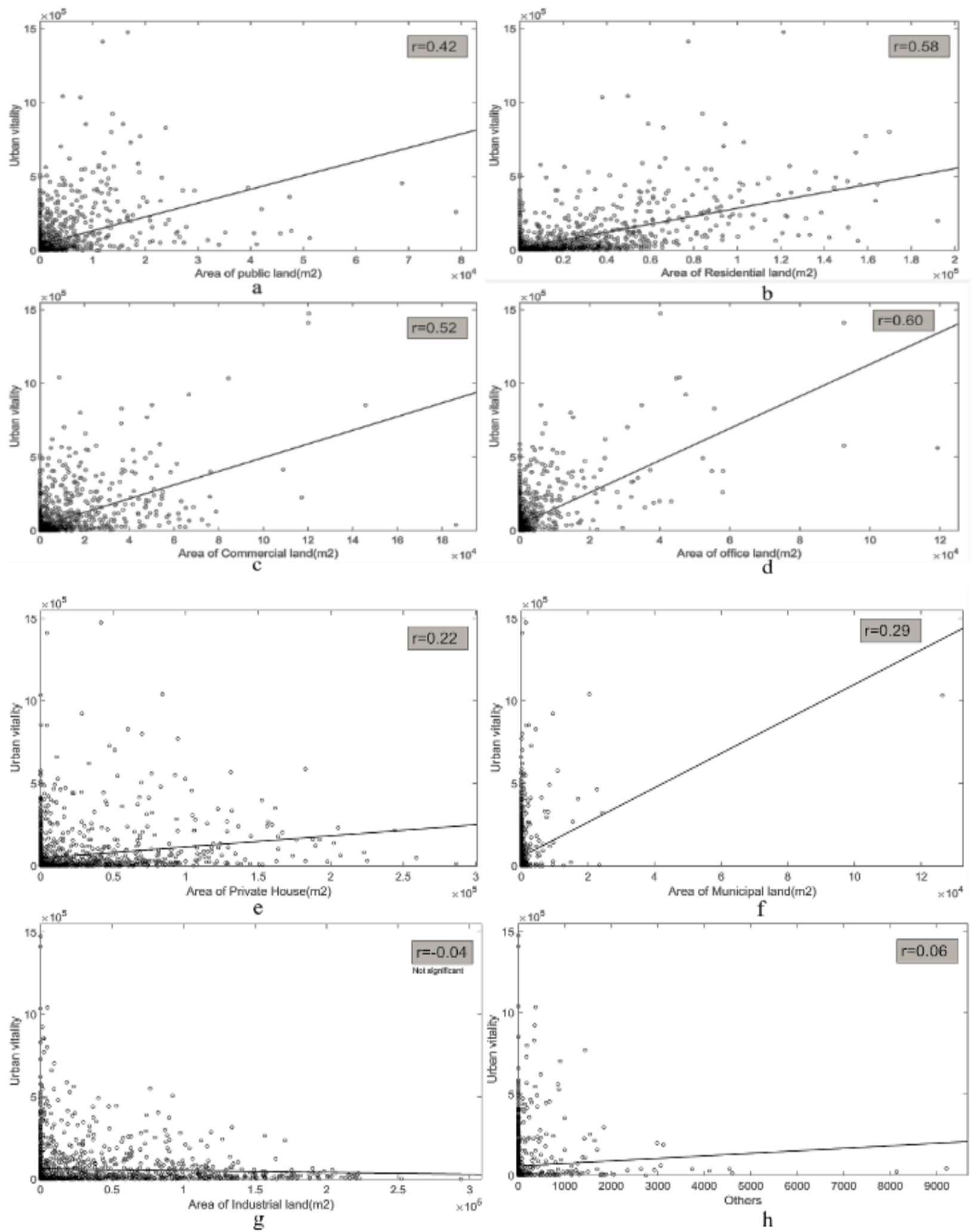

Fig. 10: Correlation between land use mixture and urban vitality 
Fig. 10: Correlation between land use mixture and urban vitality

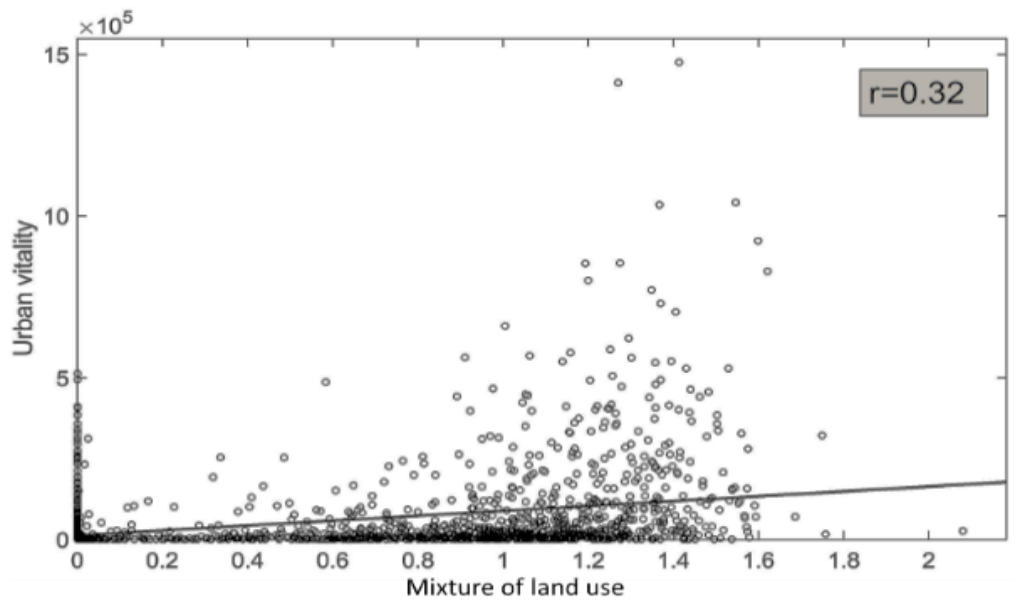

Fig. 11: Correlation between different types of land use and urban vitality
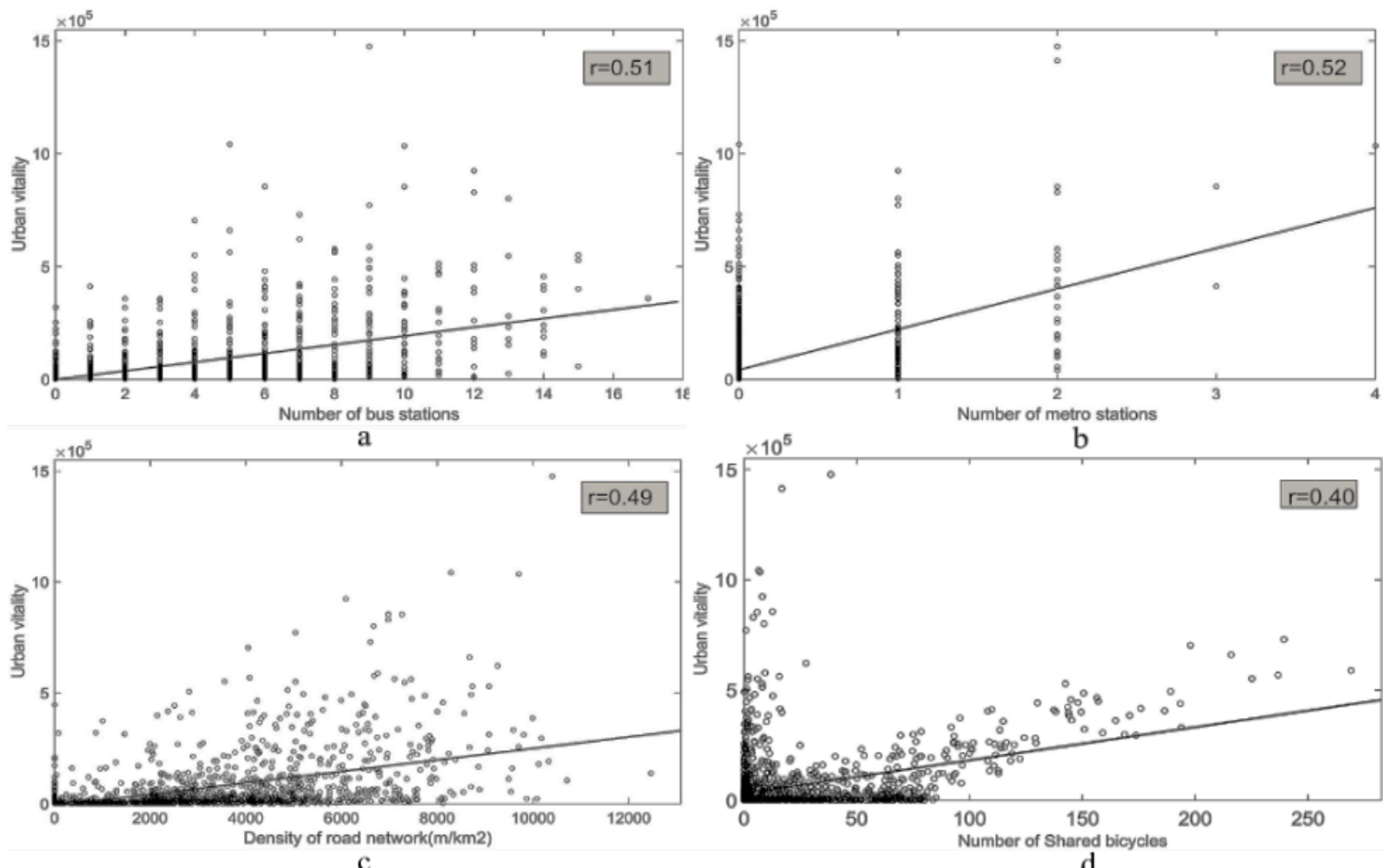

Fig. 12: Correlation between transportation facilities and urban vitality

In order to compare the influence of different variables on urban vitality, this paper first standardizes all the independent variables, the standardization method is as follows:

$$
z_{i}=\frac{x_{i}-\operatorname{mean}\left(X_{i}\right)}{\operatorname{std}\left(X_{i}\right)}
$$

As seen in Figure 13, the distribution of urban vitality is not following the normal distribution, which seems like the power law. We first need to do a logarithmic transformation on the dependent variable. After the logarithmic transformation, the dependent variable approximates the normal distribution, and then the regression model was subsequently constructed to explore the relationship between urban vitality and the built environment. 


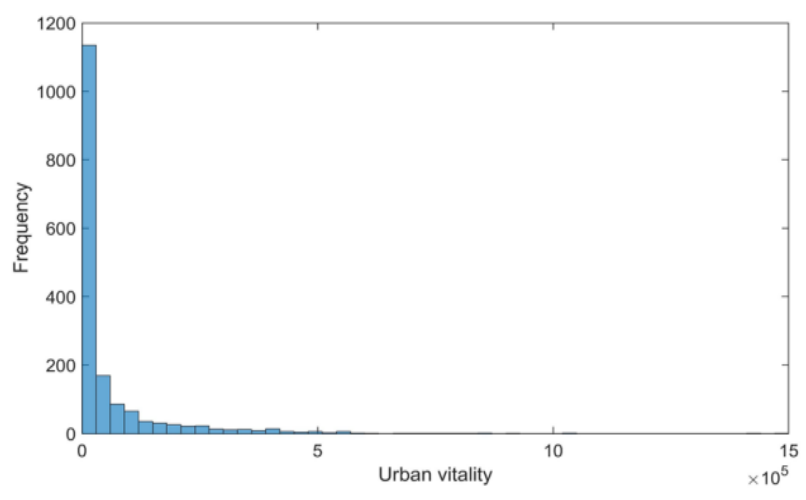

a

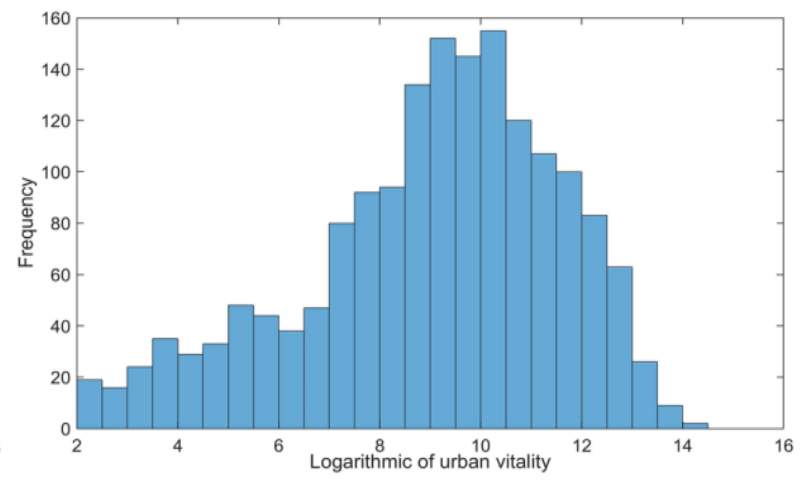

$\mathrm{b}$

Fig. 13: Histogram of urban vitality

As the regression model shows (Table 2), land use has a significant impact on urban vitality with the different effects of different land types. Commercial land can bring better urban vitality effects than residential and official land. That's because the passenger flow of residential and official land is relatively concentrated, which makes passenger flow limited during off-peak hours. On the contrary, public land has a large number of passenger flow in off-peak hours with rather limited passengers in peak hours. Industrial, municipal and urban village have no significant effect on urban vitality. The industrial land is difficult to attract enough passengers except the workers resulting in poor vitality. The municipal land is generally not a destination for residents, so it cannot make vitality. Although the urban village has a dense population, it is difficult to generate more residents' activities because of the crowded interior and poor environment.

Land use mixture has a significant effect on urban vitality. Its regression coefficient is greater than the regression coefficient of different land use types, which indicates that land use mixture has more significant effects in shaping urban vitality. This inspires us that we should strengthen the mixed development of land use and enhance the mix of functions to achieve the high vitality development of the city in the future.

A good transportation system can bring higher urban vitality. It can be seen from the regression model that there is a positive correlation between urban roads, buses, metros, and shared bicycles and urban vitality. Among them, road network density has the highest impact on urban vitality. Because high-density road network means good accessibility and pedestrian connectivity, and urban road dense areas are also better developed, which can attract more citizens. As the major public transportation tools, bus and rail transit carries a large number of residents, so areas with developed public transportation tend to have higher urban vitality. Shared bicycles are now popular with residents as a non-motorized mode of travel, which solves the problem of 'last mile travel', so sharing bicycles has a positive effect on urban vitality as well.

Table 2: Results of the regression model

\begin{tabular}{ccccc}
\hline Variable & Coefficient & Std. Error & t-Statistic & Prob. \\
\hline Constant & 9.011540 & 0.042666 & 211.2121 & $0.0000^{* * *}$ \\
Public land & 0.092153 & 0.053642 & 1.717917 & $0.0860^{*}$ \\
Office land & 0.087384 & 0.051256 & 0.924453 & $0.0881^{*}$ \\
Residential land & 0.094891 & 0.055236 & 0.234011 & $0.0866^{*}$ \\
Commercial land & 0.237262 & 0.062371 & 3.804043 & $0.0001^{* * *}$ \\
Industrial land & -0.070816 & 0.050476 & -1.402953 & 0.1608 \\
Municipal land & -0.023421 & 0.045732 & -0.512137 & 0.6086 \\
Private house & 0.047384 & 0.052744 & 0.898376 & 0.3691 \\
Others & -0.018894 & 0.043991 & -0.429494 & 0.6676 \\
Mixing degree of land & 0.347408 & 0.059412 & 5.847448 & $0.0000^{* * *}$ \\
use & & & &
\end{tabular}




\begin{tabular}{ccccc} 
Number of bus stations & 0.698781 & 0.057421 & 12.16938 & $0.0000^{* * *}$ \\
Number of metro & 0.158238 & 0.050822 & 3.113566 & $0.0019^{* * *}$ \\
stations & & & & \\
Density of network & 0.829865 & 0.055868 & 14.85413 & $0.0000^{* * *}$ \\
Number of shared & 0.158324 & 0.046209 & 3.426261 & $0.0006^{* * *}$ \\
bicycles & Adjusted R- & F-statistic & Prob(F- & AIC \\
\hline R-squared & squared & & statistic) & 3.97 \\
\hline 0.5302 & 0.5266 & 145.95 & 0.0000 & SC \\
\hline S.E. of regression & S.D. dependent & Log & Durbin- & Watson \\
\hline 1.756 & var & likelihood & 1.708583 & 4.02 \\
\hline
\end{tabular}

$* * * \leq 0.01, * * \leq 0.05, * \leq 0.1$

\section{Conclusion}

This paper analyzed the spatiotemporal characteristics of residents' travels to reveal the spatial pattern of residence, employment, transportation and entertainment in Shenzhen, based on public transportation data. The analysis suggested the most dynamic areas of Shenzhen are mainly in the core areas of Futian, Luohu and Nanshan districts with a trend of decline from the center to suburbs. Then we constructed a regression model to discuss the rules of how the built environment influences urban vitality. The result shows that both land use intensity and mixture play a positive role in urban vitality. More specifically, mixed land use is a key factor affecting the sustainability of urban vitality. Besides, there are also differences in the effects on urban vitality among different land use types as commercial land can bring better urban vitality effects than residential and official land because of the high passenger flow intensity and sustainability. A good transportation system can bring higher urban vitality. Areas with developed public transportation tend to have higher urban vitality. Road network density has the highest impact on urban vitality. Because high-density road networks means good accessibility and pedestrian connectivity, and urban road dense areas are also better developed, which can attract more citizens.

\section{References}

[1] Tridib Banerjee. The Future of Public Space: Beyond Invented Streets and Reinvented Places. Journal of the American Planning Association, 2001, 67(1):9-24.

[2] Ratti C, Frenchman D, Pulselli R M, et al. Mobile Landscapes: Using Location Data from Cell Phones for Urban Analysis. Environment \& Planning B Planning \& Design, 2006, 33(5):727-748.

[3] Schuetz T, Gascuel-Odoux C, Durand P, et al. Nitrate sinks and sources as controls of spatio-temporal water quality dynamics in an agricultural headwater catchment. Hydrology and Earth System Sciences, 2016, 20(2):843-857.

[4] Resch B, Sagl, Günther, et al. GIS-Based Planning and Modeling for Renewable Energy: Challenges and Future Research Avenues. ISPRS International Journal of Geo-Information, 2014, 3(2):662-692.

[5] Batty, M. Big data, smart cities and city planning. Dialogues in Human Geography, 2013, 3(3):274-279.

[6] Sung H, Lee S. Residential built environment and walking activity: Empirical evidence of Jane Jacobs' urban vitality. Transportation Research Part D Transport \& Environment, 2015, 41:318-329.

[7] Lunecke M G H, Mora R. The layered city: pedestrian networks in downtown Santiago and their impact on urban vitality. Journal of Urban Design, 2017:1-18.

[8] Jacobs J. The Death and Life of Great American Cities. Random House LLC, 1961.

[9] Wirth L. Urbanism as a Way of Life [M]// Soziologische Forschung in Unserer Zeit. VS Verlag für Sozialwissenschaften, 1951.

[10] Brenner N, Elden S. Henri Lefebvre on State, Space, Territory [J]. International Political Sociology, 2009, 3(4):353- 
377.

[11] Salehi J, Haghifam M R. Long term distribution network planning considering urbanity uncertainties [J]. International Journal of Electrical Power \& Energy Systems, 2012, 42(1):321-333.

[12] Jabareen, Y. R. Sustainable Urban Forms: Their Typologies, Models, and Concepts. Journal of Planning Education and Research, 2006, 26(1):38-52.

[13] Oliveira, V., \& Medeiros, V. (2016). Morpho: Combining morphological measures. Environment and Planning B: Planning and Design, 43(5), 805-825.

[14] Banerjee, Tridib. The Future of Public Space: Beyond Invented Streets and Reinvented Places. Journal of the American Planning Association, 2001, 67(1):9-24.

[15] Delclòs-Alió, Xavier, Miralles-Guasch C. Looking at Barcelona through Jane Jacobs's eyes: Mapping the basic conditions for urban vitality in a Mediterranean conurbation. Land Use Policy, 2018, 75:505-517.

[16] Xun Z, Wei W. Analysis on urban vitality elements and model construction. International Conference on Electric Technology \& Civil Engineering. 2011.

[17] Rowley, Alan. Definitions of urban design: The nature and concerns of urban design. Planning Practice and Research, 9(3), 179-197.

[18] Zhou X, Chen H . Impact of urbanization-related land use land cover changes and urban morphology changes on the urban heat island phenomenon. The Science of the Total Environment, 2018, 635(SEP.1):1467-1476.

[19] Lunecke M G H, Mora R. The layered city: pedestrian networks in downtown Santiago and their impact on urban vitality. Journal of Urban Design, 23(3), 336-353.

[20] Ivor, Samuels. ISUF Task Force on Research and Practice in Urban Morphology: an Interim Report. Urban morphology, 2013.

[21] Liu, Xingjian, Song, Yan, Wu, Kang, Wang, Jianghao, Li, Dong, \& Long, Y. (2015). Understanding urban China with open data. Cities, 47, 53-61.

[22] Haklay M. How good is volunteered geographical information? A comparative study of Open Street Map and Ordnance Survey datasets. Environment and Planning B: Planning and Design, 2010, 93(4):3-11.

[23] Cheng Z, Caverlee J, Kamath K Y, et al. [ACM Press the 20th ACM international conference - Glasgow, Scotland, UK (2011.10.24-2011.10.28)] Proceedings of the 20th ACM international conference on Information and knowledge management-CIKM \"11-Toward traffic-driven location-based web search. 2011:805.

[24] Frank L D , Sallis J F , Conway T L, et al. Many Pathways from Land Use to Health: Associations between Neighborhood Walkability and Active Transportation, Body Mass Index, and Air Quality. Journal of the American Planning Association, 2006, 72(1):75-87.

[25] Forsyth, A., Oakes, J.M., Schmitz, K.H., Hearst, M., 2007. Does residential density increase walking and other physical activity? .Urban Study. 44 (4), 679-697.

[26] Boarnet M G, Forsyth A, Day K, et al. The Street Level Built Environment and Physical Activity and Walking: Results of a Predictive Validity Study for the Irvine Minnesota Inventory. Environment \& Behavior, 2011, 43(6): 735-775.

[27] Park S H, Kim J H, Choi Y M, et al. Design elements to improve pleasantness, vitality, safety, and complexity of the pedestrian environment: evidence from a Korean neighborhood walkability case study. International journal of urban sciences, 2013, 17(1):142-160.

[28] Lovasi G S, MPH, Grady S, et al. Steps Forward: Review and Recommendations for Research on Walkability, Physical Activity and Cardiovascular Health. Public Health Reviews, 2011, 33(2):484-506.

[29] Sallis J F, Floyd M F, et al. Role of Built Environments in Physical Activity, Obesity, and Cardiovascular Disease. Circulation, 2012, 125(5):729-737.

[30] Koohsari M J, Sugiyama T, et al. Street connectivity and walking for transport: Role of neighborhood destinations. Preventive Medicine, 2014, 66:118-122.

[31] Sung H G, Go D H, Choi C G. Evidence of Jacobs's street life in the great Seoul city: Identifying the association of physical environment with walking activity on streets. Cities, 2013, 35:164-173. 
[32] Shewing H E, Liu Y, Fulong W U, et al. Social Groups and Housing Differentiation in China's Urban Villages: An Institutional Interpretation. Housing studies, 2010, 25(5):P.671-691.

[33] Liu, Xingjian, Song, Yan, Wu, Kang, Wang, Jianghao, Li, Dong, \& Long, Y. (2015). Understanding urban China with open data. Cities, 47, 53-61.

[34] Ye Y, Li D, Liu X. How block density and typology affect urban vitality: an exploratory analysis in Shenzhen, China. Urban Geography, 2017:1-22. 